\title{
Influência do escore de condição corporal na probabilidade de prenhez em bovinos de corte
}

\author{
Torres, H.A.L. ${ }^{\text {; }}$ Tineo $^{2}$, J.S.A. e Raidan, F.S.S. ${ }^{2 @}$
}

'Instituto de Ciências Agrárias da Universidade Federal de Minas Gerais (ICA/UFMG). Montes Claros. MG. Brasil. ${ }^{2}$ Escola de Veterinária da Universidade Federal de Minas Gerais (EV/UFMG). Belo Horizonte. MG. Brasil.

\section{PALAVRAS CHAVE ADICIONAIS}

Manejo reprodutivo.

Nelore.

Inseminaç̃ão artificial em tempo fixo.

\section{ADDITIONAL KEYWORDS \\ Reproductive management. \\ Nellore. \\ Timed artificial insemination.}

\begin{abstract}
RESUMO
Objetivou-se avaliar a influência do escore de condição corporal (ECC) na probabilidade de prenhez de fêmeas Nelore em programas de inseminação artificial em tempo fixo (IATF). Realizou-se o protocolo de IATF e a avaliação visual do ECC (escala biológica de 1, muito magra a 5, muito gorda) em 5.082 fêmeas de diferentes ordens de parto criadas em três fazendas localizadas na região Norte de Minas Gerais. O resultado do diagnóstico de gestação - positivo, 1 e negativo, 0 foi utilizado para estudo da probabilidade de prenhez, modelada por meio de regressão logística, a partir das variáveis regressoras: ordens de parto, fazenda e escore de condição corporal. A probabilidade de prenhez média foi $52,03 \%$. As variáveis fazenda e ordem de parto não influenciaram a prenhez das fêmeas Nelore. $\bigcirc$ aumento em 0,5 unidade de ECC implicou um incremento de 39,0\% na probabilidade de prenhez. $\mathrm{O}$ escore de condição corporal interfere na probabilidade de prenhez de fêmeas bovinas da raça Nelore em programas de inseminação artificial em tempo fixo.
\end{abstract}

Influence of body condition score in pregnancy probability in beef cattle

SUMMARY

This study aimed to evaluate the influence of body condition score (BCS) on the probability of pregnancy in Nellore cattle on programs of timed artificial insemination (TAI). The TAI protocol and visual evaluation of the BCS (being 1, too lean to 5, too fat) were developed on 5,082 females with different calve orders raised in three farms located in Northern Minas Gerais. The result of pregnancy diagnosis -positive, 1 and negative, 0 was used to study the probability of pregnancy, from the model of logistic regression, from the regression variable as calve orders, farm and body conditions. The mean of probability of pregnancy was $52.03 \%$. The farm and calve orders effect did not influence the pregnancy probability in Nellore cattle. An increase of 0.5 unit of BC showed an increase of $39.0 \%$ in the pregnancy probability. The body condition affects the probability of pregnancy in Nellore cows in timed artificial insemination programs.

\section{INFORMACIÓN}

Cronología del artículo.

Recibido/Received: 3.3.2015

Aceptado/Accepted: 16.7.2015

On-line: 16.9.2015

Correspondencia a los autores/Contact e-mail:

fernandaraidan@gmail.br

\section{INTRODUÇÃO}

A atual demanda mundial pela produção de carne bovina para consumo humano exige do produtor grande esforço para a melhoria dos indicadores de eficiência reprodutiva e produtiva de seus rebanhos, com vistas à melhoria da taxa de desfrute e consequente retorno econômico da atividade (Ferreira et al., 2013). A eficiência produtiva é primordial para a lucratividade da pecuária de corte e o aumento da produção, em $\mathrm{kg}$ de peso vivo total ou $\mathrm{kg}$ de peso vivo/ha/ano, depende dos índices reprodutivos do rebanho (Barbosa et al., 2010). Neste contexto, a sincronização da ovulação que possibilita o emprego da inseminação artificial em tempo fixo (IATF) pode ser utilizada como ferramenta de manejo reprodutivo de bovinos de corte auxiliando na melhoria dos indicadores reprodutivos como taxa de prenhez e de natalidade. Nos sistemas de cria esses índices são variáveis de maior impacto na avaliação do desempenho reprodutivo e contribuem consideravelmente para a eficiência bioeconômica (Barcellos et al., 2006). Além disso, o uso da IATF pode facilitar o manejo reprodutivo nos sistemas de produção de bovinos de corte, por permitir concentrar as atividades relacionadas à observação do estro e diagnóstico de gestação. Contudo, o planejamento nutricional é fator fundamental para maximização dos resultados a serem obtidos com a IATF. 
O sucesso nos programas de IATF está relacionado à eficiência do manejo alimentar do rebanho e ao atendimento das exigências nutricionais das vacas nos períodos pré e pós-parto. Assim, poderá ocorrer a ativação de processos fisiológicos relacionados à reprodução e o incremento no peso corporal. Alternativa prática e de baixo custo de implantação, para monitorar o estado nutricional e o desempenho reprodutivo de matrizes é a avaliação visual do escore de condição corporal (ECC, Richard et al., 1986). Segundo Sonohata et al. (2009) o ECC é fator determinante das taxas reprodutivas e esta, por sua vez, varia em função do manejo nutricional, sanitário e composição genética dos rebanhos.

O monitoramento do ECC em animais com idade reprodutiva pode contribuir para aumento na taxa de prenhez dos rebanhos (Ferreira et al., 2013) e no desenvolvimento de ações para otimizar o número de fêmeas gestantes ao final da estação reprodutiva. Dessa forma, objetivou-se avaliar a influencia do escore de condição corporal na probabilidade de prenhez de fêmeas Nelore em programas de inseminação artificial em tempo fixo.

\section{MATERIAL E MÉTODOS}

Um experimento com 5.082 fêmeas Nelore foi realizado na região Norte de Minas Gerais, sendo 1.492 animais criados na Fazenda Capim Verde, 2.570 na Fazenda Fortaleza e 1.020 na Fazenda Veredinha, localizadas nos municípios de Jaíba, Jequitaí e Itacarambi, respectivamente. Essa região apresenta clima, segundo classificação de Köppen, do tipo Aw, caracterizado por inverno seco e verão chuvoso, precipitação média anual de $800 \mathrm{~mm}$, irregularmente distribuída de novembro a abril, com temperatura média anual de $26^{\circ} \mathrm{C}$ e máxima de $40^{\circ} \mathrm{C}$ (Brasil, 1992).

Os animais avaliados foram classificados de acordo com as ordens de parto em nulíparas, primíparas e secundíparas e foram submetidos a sistema de criação extensivo em pastagens. Durante os meses de verão, de novembro a abril, os animais receberam volumoso via pastagens, predominantemente Brachiaria decumbens e pequenas aéreas de Brachiaria brizantha e Cenchrus ciliares e suplementação mineral. Nos meses de inverno, de maio a outubro, além da fonte de volumoso e mineral foi fornecida suplementação protéico-energética de acordo com a fase de crescimento. A avaliação visual de ECC foi realizada por um único avaliador segundo a metodologia descrita por Houghton et al. (1990), adaptada para intervalos de 0,25. A avaliação de ECC ocorreu no início do procedimento de sincronização do cio por meio de exame visual, considerando a escala biológica de um (muito magra) a cinco (muito gorda).

Os animais foram submetidos ao protocolo de IATF realizado entre os meses de janeiro a março de 2013. Foram utilizadas no experimento as fêmeas com atividade ovariana cíclica normal, ou seja, aquelas que no exame clínico (realizado com ultrassonografia) apresentavam presença de corpo lúteo (CL) no dia 0. Após avaliação dos ovários foi realizada a aplicação de 2,0 mg de benzoato de estradiol (2,0 mL de Estrogin $\left.{ }^{\circledR}\right)$ e inserção do dispositivo intravaginal de progesterona $\mathrm{CIDR}^{\circledR}(1,9 \mathrm{~g}$ de progesterona). No dia 7 os animais receberam $12,5 \mathrm{mg}$ de prostaglandina F2 alfa - PGF2 $\alpha$, (2,5 mL de Lutalyse $\left.{ }^{\circledR}\right)$. No dia 9 foi removido o dispositivo de progesterona, os bezerros foram separados de suas mães e foi aplicado 0,6 $\mathrm{mg}$ de cipionato de estradiol $\left(0,30 \mathrm{~mL}\right.$ de E.C.P. $\left.{ }^{\circledR}\right)$ em todos os animais. Adicionalmente em nulíparas foram aplicadas 200 UI de Gonodotrofina Coriônica Equina (eCG, Pregnant Mare Serum Gonadotrophin - PMSG), equivalentes a 1 $\mathrm{mL}$ de Novormon ${ }^{\circledR}$. No dia $11,48 \mathrm{~h}$ após a retirada do dispositivo de progesterona, todos os animais foram inseminados artificialmente e os bezerros retornaram ao aleitamento com suas mães (figura 1).

O resultado do diagnóstico de gestação (sucesso $=1$ e insucesso $=0$ ), obtido 30 dias após a inseminação artificial, foi utilizado para estudo da probabilidade de prenhez que foi modelada por meio de regressão logística, considerando-se como variáveis regressoras o escore de condição corporal, a ordem de parto e a fazenda de criação dos animais de acordo com o modelo geral:

$$
\ln \left(\frac{P_{i j k l}}{1-P_{i j k l}}\right)=\beta_{0}+\beta_{1} x_{1 i}+\beta_{2} x_{2 j}+\beta_{3} x_{3 k}+\varepsilon_{i j k l} ;
$$

em que: $P_{i j k l}$ é a probabilidade de prenhez do animal 1 , com ordem de parto $i\left(x_{1 i}\right)$, criado na fazenda $j\left(x_{2 j}\right)$ e escore de condição corporal $k\left(x_{3 k}\right)$; é uma constante geral presente em todas as observações; $\beta_{h}(\mathrm{~h}=1,2$ e 3 ) são os coeficientes de regressão associados as variáveis regressoras $X_{h}$; e $\varepsilon_{i j k l}$ é o erro aleatório associado a cada observação.

As estimativas dos parâmetros $\left(\hat{\beta}_{h}\right)$ foram obtidas pelo método da máxima verossimilhança e o modelo final foi escolhido por meio da opção Stepwise do procedimento Logistic do Statistical Analysis System - SAS $^{\circledR}$ (SAS, 2003).

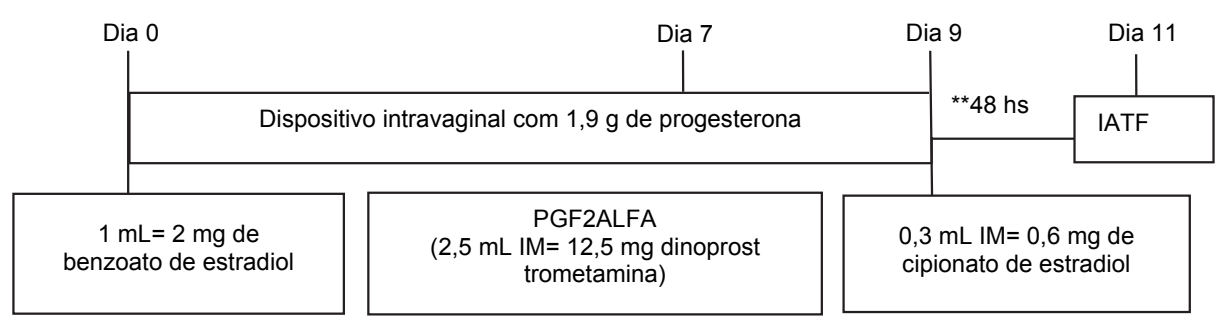

Figura 1. Protocolo para inseminação artificial em tempo fixo (IATF) em fêmeas Nelore (Protocol for artificial fixed time insemination (AFT) in Nellore). 
Tabela I. Estimativa de parâmetros ${ }^{1}$ para escore de condição corporal (ECC) na modelagem da probabilidade de prenhez de fêmeas Nelore em programas de inseminação artificial em tempo fixo (Estimate parameters ${ }^{1}$ for body condition score (BCS) in modeling the probability of pregnancy in Nellore females artificial insemination programs at fixed time).

\begin{tabular}{|c|c|c|c|c|c|c|c|c|}
\hline \multirow[b]{2}{*}{ Variável regressora } & \multicolumn{2}{|c|}{ Coeficiente de regressão } & \multirow{2}{*}{ Odds ratio } & \multirow{2}{*}{ IC } & \multicolumn{2}{|c|}{$\%$ pares } & \multirow{2}{*}{ Suc/lns } & \multirow[b]{2}{*}{$\mathrm{p}$} \\
\hline & $\hat{\beta}_{0}$ & $\hat{\beta}_{0}$ & & & Conc. & Disc. & & \\
\hline Ordem & & $-0,0340$ & 0,967 & 0,$897 ; 1,037$ & & & & 0,3199 \\
\hline Fazenda & $-1,2702$ & $-0,0361$ & 0,965 & 0,$904 ; 1,034$ & 50,3 & 42,3 & $2643 / 2437$ & 0,3278 \\
\hline ECC & & 0,5825 & 1,790 & 1,$469 ; 2,182$ & & & & $<0,0001$ \\
\hline
\end{tabular}

${ }^{1}$ Suc/Ins= Sucesso/insucesso; Odds ratio= Razão entre chances; IC= Intervalo de confiança; Conc.= Concordantes; Disc.= Discordantes.

A odds ratio (p(sucesso)/p(insucesso)) associada a cada variável regressora foi estimada por meio de $\mathrm{e}^{\hat{\hat{\beta}_{h}}} \mathrm{e}$ define a mudança na odds ratio quando houver alteração de uma unidade da variável regressora (Freund and Littell, 2000).

A estatística utilizada para aferir a validade dos modelos foi à porcentagem de pares de observaçõespredições concordantes. Esse procedimento consiste em comparar as probabilidades entre as respostas observadas e preditas considerando todos os possíveis pares de observações $\left(y_{i}, y_{j}\right.$ onde $\left.i \neq j\right)$, de modo que as respostas observadas para $y_{i}^{\prime} s$ sejam iguais a um (sucesso) e as observadas para $y_{j}^{\prime}$ s sejam iguais a zero (insucesso). Dessa forma, houve a formação de $n_{i} \times n_{j}$ pares de observações (sucesso $\times$ insucesso no diagnóstico de gestação) que foram classificados em concordantes quando $\hat{P}_{i}>\hat{P}_{j}$, discordante quando $\hat{P}_{i}<\hat{P}_{j}$, e empatados se $\hat{P}_{i}=\hat{P}_{j}$. A proporção para cada modelo foi calculada em relação ao total de pares $n_{i} \times n_{j}$ (Bergmann and Hohenboken, 1992).

O nível de significância para os efeitos das fontes de variação foi calculado pelo teste da razão de verossimilhança, que consiste da comparação entre o valor da função de verossimilhança obtida no modelo reduzido, que considera apenas a constante, e o valor da função no modelo completo, que inclui a variável regressora além do intercepto.

Para facilitar a interpretação dos efeitos de cada variável regressora incluída no modelo final separadamente foram construídos gráficos a partir dos valores de $\hat{\beta}_{h}$ mantendo-se $x_{h}=x_{h}$ e variando-o dentro da amplitude observada para a variável regressora de interesse.

\section{RESULTADOS E DISCUSSÃO}

A média \pm desvio-padrão para escore de condição corporal foi de $2,48 \pm 0,28$ e essa variável apresentou baixa instabilidade, com coeficiente de variação de $11,29 \%$ e valores mínimos e máximos de 2 e 4 , respectivamente. Ferreira et al. (2013) afirmaram que fêmeas com ECC superior a 2 (escala de 1 a 5 ) tem condições fisiológicas adequadas para manter a atividade cíclica normal. Sonohata et al. (2009) observaram que fêmeas Nelore criadas na sub-região da Nhecolândia no Pantanal Sul-Matogrossense com ECC igual ou superior a 2,25 apresentaram atividade cíclica normal. Barbosa et al. (2011) também observaram que ECC mínimo de 2,25 implica em atividade cíclica normal para fêmeas leiteiras mestiças Bos taurus $\times$ Bos indicus. Dessa forma, o ECC observado no presente estudo pode ser conside- rado como adequado para garantir a atividade cíclica de fêmeas da raça Nelore.

A taxa de prenhez média foi de $52,03 \%$, sendo similar aos valores de $61,81 \%, 51,51 \%$ e $57,41 \%$ descritos por Sales et al. (2014), para fêmeas Nelore com ECC médio de $3 \pm 0,50$ submetidas a programas de IATF e criadas em três unidades produtivas em sistemas extensivos no Norte de Minas Gerais. Batista et al. (2012) apresentaram taxas de prenhez média para fêmeas $\mathrm{Ne}$ lore de $35,29 \%, 40,98 \%, 74,14 \%$ e $52,00 \%$ em estações de inseminação realizadas nos anos de 2006, 2007, 2008 e 2009, respectivamente. Esses autores não utilizaram protocolo de inseminação artificial em tempo fixo e afirmaram que a não-detecção do estro, inibição social, altas temperaturas e déficit hídrico podem ser responsáveis pelas baixas taxas verificadas nos anos de 2006 e 2007, a maior média observada no ano de 2008 foi relacionada à substituição de pluríparas por nulíparas.

O efeito da fazenda $(p=0,3278)$ não foi significativo sobre a probabilidade de prenhez de fêmeas Nelore (tabela I) possivelmente como consequência da similaridade de manejo adotado pelas três fazendas avaliadas, com alimentação baseada em pastagens e suplementação mineral, nos meses de verão e adição de suplementação protéico-energética nos meses de inverno.

O efeito de ordem de parto $(p=0,3199)$ não influenciou a probabilidade de prenhez (tabela I). No entanto, Batista et al. (2012) observaram maiores taxas de prenhez para nulíparas $(84 \%)$ quando comparadas a primíparas $(43 \%)$ e multíparas $(47 \%)$. Esses autores afirmaram que multíparas e primíparas são mais dependentes do ambiente a que estão submetidas, principalmente ao manejo alimentar, quanto comparadas as nulíparas (Batista et al., 2012). Sonohata et al. (2009) observaram efeito significativo para interação entre ECC e idade de vaca, isso evidenciou que vacas mais jovens são mais dependentes da condição corporal para emprenhar do que vacas mais velhas. Menores taxas de prenhez para primíparas pode esta relacionado ao estresse ao parto, e aos efeitos combinados entre o crescimento e a primeira lactação, fazendo com que os requisitos nutricionais se elevem, resultando em baixa resposta reprodutiva (Spitzer et al., 1995). Segundo Short et al. (1980) a característica produtiva mais sensível de uma vaca em reprodução é a taxa de reconcepção, pois é a última a receber energia disponível, que atende a seguinte ordem de importância: metabolismo basal, atividade, crescimento, reservas de energia, prenhez, lactação, reservas adicionais de energia, ciclo 


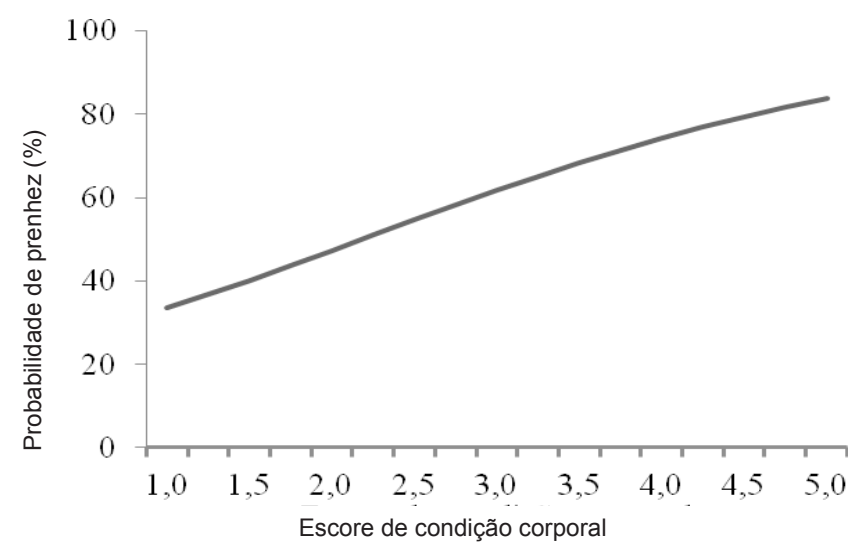

Figura 2. Probabilidade de prenhez em função do escore de condição corporal para fêmeas bovinas da raça Nelore submetidas a programas de inseminação artificial em tempo fixo (Pregnancy probability as a function of body condition score for Nellore cows from undergoing artificial insemination programs at fixed time).

estral e início de prenhez e reservas de energia em excesso. $\mathrm{O}$ atendimento as exigências nutricionais de cada categoria animal é fator determinante dos índices reprodutivos dos rebanhos (Short et al., 1980) e pode reduzir diferenças relativas a ordem de parto e permitir que os animais expressem seu potencial genético para produção e reprodução. Dessa forma, é possível inferir que no presente estudo o manejo nutricional foi adequado e por isso a ordem de parto não influenciou o escore de condição corporal.

O ECC $(p<0,001)$ influenciou a probabilidade de prenhez de Fêmeas Nelore (tabela I). A odds ratio estimada foi de 1,78, indicando que o aumento em 0,5 unidades de ECC implicaria um aumento de 39\% na probabilidade de prenhez dos animais. Grecellé et al. (2006) também observaram efeito significativo entre ECC e probabilidade de prenhez. De forma similar ao encontrado nesse estudo esses autores afirmaram que o aumento de 0,5 unidades no ECC elevaria em 34,1\% a probabilidade de prenhez. Ferreira et al. (2013) observaram $86,5 \%$ de prenhez em pluríparas da raça Nelore com ECC entre 3,0 e 4,0 e 65,9\% para animais com ECC entre 2,0 e 2,5\%, superiores às médias observadas neste estudo (52,03\%). No entanto, Ferreira et al. (2013) afirmaram que essas matrizes foram expostas aos touros de 24 horas após a IATF até o final da estação de monta e esse procedimento pode influenciar nos resultados.

Santos et al. (2009) e Sonohata et al. (2009) também observaram maior taxas de prenhez em fêmeas com maior ECC e afirmaram que a seleção de vacas adaptadas as condições edafoclimáticas juntamente com o desenvolvimento de planos nutricionais adequados possibilitam melhoria nos índices reprodutivos de sistemas de produção de bovinos de corte. No entanto, Meneguetthi et al. (2008) não observaram influencia do ECC na probabilidade de prenhez de fêmeas Nelore ou $1 / 2$ Nelore + 1/2 Red Angus. A aplicação de estradiol no final de protocolo de IATF, na retirada do CIDR $^{\circledR}$, pode resultar em efeito positivo sobre a motilidade uterina e o transporte espermático através do trato reprodutivo, por aumentar os níveis circulantes de estradiol, que estariam mais baixos nas vacas de menor ECC devido à reduzida capacidade esteroidogênica de seus folículos pré-ovulatórios (Meneguetthi et al., 2008). Isso explicaria a similaridade na taxa de prenhez das vacas com diferentes ECC obtidas por Meneguetthi et al. (2008).

A maior probabilidade de prenhez foi observada para fêmeas ECC igual a 5,0 (figura 2), no entanto, animais com alto ECC podem apresentar irregularidades no ciclo estral decorrente do acúmulo de gordura nos órgãos reprodutivos (Duarte Júnior et al., 2013). Além disso, fêmeas nessas condições apresentam maiores riscos de aborto, partos distócicos, baixa produção de leite (Fuck et al., 2000) e tendências a distúrbios metabólicos. Em programas de inseminação artificial em tempo fixo a prioridade de uso seria para fêmeas com ECC de 3,5 $\pm 0,5$. Isso resultaria em taxa de prenhez superior a $60,0 \%$ em função do maior número de animais com atividade cíclica normal e peso mínimo critico. Os resultados obtidos neste estudo corroboram aqueles encontrados por Gottschall et al. (2008), Ferreira et al. (2013) e Sales et al. (2014). Dessa forma, a avaliação visual de ECC pode fornecer subsídios para auxiliar a formação de lotes de manejo, definir estratégias de suplementação nutricional e, consequentemente, melhorar os índices reprodutivos dos rebanhos.

\section{CONCLUSÕES}

A avaliação visual da condição corporal pode ser utilizado como ferramenta auxiliar no manejo reprodutivo de bovinos de corte.

O escore de condição corporal interefere na probabilidade de prenhez de fêmeas bovinas da raça Nelore e animais com escore de condição corporal superior a 3,0 proporcionam maiores taxas de prenhez em programas de inseminação artificial em tempo fixo.

\section{AGRADECIMENTOS}

Os autores agradecem aos produtores pela disponibilização dos dados, a empresa Biocampo pela parceria e a Capes, CNPq, FAPEMIG e Universidade Federal de Minas Gerais pelo apoio financeiro.

\section{BIBLIOGRAFIA}

Barbosa, C.F.; Jacomini, J.O.; Diniz, E.G.; Santos, R.M. e Tavares, M. 2011 . Inseminação artificial em tempo fixo e diagnóstico precoce de gestação em vacas leiteiras mestiças. Rev Bras Zootec, 40: 79-84.

Barbosa, F.A.; Graça, D.S.; Andrade, V.J.; Cezar, I.M.; Santos, G.G. e Souza, R.C. 2010. Produtividade e eficiência econômica de sistemas de produção de cria, recria e engorda de bovinos de corte na região sul do estado da Bahia. Arq Bras Med Vet Zoo, 62: 677-685.

Barcellos, J.O.J.; Silva, M.D.; Prates, E.R. e Costa, E.C. 2006. Taxas de prenhez em novilhas de corte acasaladas aos 18 e 24 meses de idade. Arq Bras Med Vet Zoo, 58: $1168-1173$.

Batista, D.S.N.; Abreu, U.G.P., Ferraz Filho, P.B. e Rosa, A.N. 2012. Índices reprodutivos do rebanho Nelore da fazenda Nhumirim, Pantanal da Nhecolândia. Acta Sci, 34: 71-76.

Bergmann, J.A.G. and Hohenboken, W.D. 1992. Prediction of fertility from calfhood traits of Angus and Simenthal heifers. J Anim Sci, 70: 2611-2621.

Brasil. Ministério da Agricultura e Reforma Agrária. Secretaria Nacional de Irrigação. Departamento Nacional de Meteorologia. 1992. Normas climatológicas: 1961-1990. Brasília. 84 pp. 
Duarte Júnior, M.F.; Hatamoto-Zervoudakis, L.K.; Zervoudakis, J.T.; Kocheck, J.F.W.; Fioravanti Filho, R.S. e Freitas, L.C. 2013. Aspectos relacionados à fisiologia do anestro pós-parto em bovinos. Colloquium Agrariae, 9: 43-71.

Ferreira, M.C.N.; Miranda, R.R.; Figueiredo, M.A.; Costa, O.M. e Palhano, H.B. 2013. Impacto da condição corporal sobre a taxa de prenhez de vacas da raça nelore sob regime de pasto em programa de inseminação artificial em tempo fixo (IATF). Semin Cienc Agrar, 34: 1861-1868.

Freund, R.J. and Littell, R.C. SAS ${ }^{\circledR}$ System for regression. SAS Institute, 2000. $3^{a}$ ed. SAS Institute Inc, Cary, NC. 235 pp.

Fuck, E.J.; Moraes, G.V. e Santos, G.T. 2000. Fatores nutricionais na reprodução das vacas leiteiras. I. Energia e proteína. Rev Bras Reprod Ani, 24: 147-161.

Grecellé, R.A.; Barcellos, J.O.J.; Braccini Neto, J.; Costa, E.C. e Prates, E.R. 2006. Taxa de prenhez de vacas Nelore $x$ Hereford em ambiente subtropical sob restrição alimentar. Rev Bras Zootec, 35: 1423-1430.

Gottschall, C.S.; Marques, P.R.; Canellas, L. e Almeida, M.R. 2008. Aspectos relacionados à sicronização do estro e ovulação em bovinos de corte. A Hora Vet, 164: 43-48.

Houghton, P.L.; Lemenager, R.P.; Moss, G.E. and Hendrix, K.S. 1990. Prediction of postpartum beef cow body composition using weight to height ratio and visual body condition score. JAnim Sci, 68: 1428-1437.

Meneguetti, M. e Vasconcelos, J.L.M. 2008. Mês de parição, condição corporal e resposta ao protocolo de inseminação artificial em tempo fixo em vacas de corte primíparas. Arq Bras Med Vet Zootec, 60: 786-793.
Richards, M.W.; Spitzer, J.C. and Warner, M.B. 1986. Effect of varying levels of postpartum nutrition and body condition at calving on subsequent reproductive performance in beef cattle. JAnim Sci, 62: 300-306.

Sales, L.H.B.; Rebello, R.V.; Soares, A.C.M.; Glória, J.R. e Oliveira, N.J.F. 2014. Separação fenotípica e taxas de prenhez após inseminação Artificial em tempo fixo em bovinos de corte. Enciclop Bios, 10: $2757-2766$

Santos, S.A.; Abreu, U.G.P.; Souza, G.S. e Catto, J.B. 2009. Condição corporal, variação de peso e desempenho reprodutivo de vacas de cria em pastagem nativa no Pantanal. Rev Bras Zootecn, 38: 354-360.

SAS. 2003. Statistical Analysis System. User's guide. Version 9. 1. Statistical Analysis System Institute. Cary, NC.

Short, R.E.; Bellows, R.A.; Staigmiller, R.B.; Berardinelli, J.G. and Custer, E.E. 1990. Physiological mechanisms controlling anestrus and infertility in postpartum beef cattle. J Anim Sci, 68: 799-816.

Sonohata, M.M.; Oliveira, C.A.L.; Canuto, N.G.D.; Abreu, U.G.P. e Fernandes, D.D. 2009. Escore de condição corporal e desempenho reprodutivo de vacas no Pantanal do Mato Grosso do Sul. Brasil. Rev Bras Sau Prod Anim, 10: 988-998.

Spitzer, J.C.; Morrison, D.G.; Wettemann, R.P. and Faulkner, C.C. 1995. Reproductive responses and calf birth and weaning weights as affected by body condition at parturition and postpartum weight gain in primiparous beef cows. J Anim Sci, 73: 1251-1257. 
